\title{
Sensory-Motor Coupling in Rehabilitation Robotics
}

\author{
Alejandro Hernandez-Arieta, Konstantinos Dermitzakis, Dana Damian, \\ Max Lungarella and Rolf Pfeifer \\ University of Zurich, Artificial Intelligence Laboratory, Switzerland
}

\section{Introduction}

The general well-being of people has always been a strong drive towards the improvement of available technologies and the development of new ones. Recently, a greater longevity and the consequent increase of physically challenged elder adults have increased the significance of research on assistive technologies such as rehabilitation robots, power-assist systems, and prosthetic devices. One important goal of these research endeavors is the restoration of lost motor function for people with disabilities (e.g. locomotion, manipulation, and prehension) with prostheses such as robot hands, arms, ankles, and legs (Lebedev et al., 2006; Kato et al., 2006). Although such prosthetic devices are increasingly more intuitive to use and yield better functionality, most users still fail to recognize the prosthesis as an integral part of their body - a problem akin to the one affecting people with lesions to their afferent nervous system (Fig. 1) (Tsakiris et al., 2007). Such lack of integration makes the control of the prosthetic device cumbersome, and thus leads to excessive and unwanted cognitive load (Hunter et al., 2003). To counter this problem, some prosthetic applications attempt to strengthen the user-environment coupling (Pylatiuk et al, 2006), for example by feeding back visual or vibro-tactile stimuli to the user.

Clearly, one important challenge of rehabilitation robotics is how to "trick" the user of the prosthesis to accept the device as an integral part of their body. There are at least three ways to tackle this challenge: (1) Exploit the trends in information technology such as more energy-efficient and powerful microcontrollers, allowing faster sensory feedback and better real-time performance at a smaller scale. (2) Use smart materials and adaptive mechanisms to reduce computational complexity and off-load more to the morphological properties of the prosthetic device (Dollar \& Howe, 2007; Pfeifer et al., 2006; Pfeifer et al., 2007). (3) Improve human-machine interaction by developing "intelligent" machines that can guess the user's intention and are able to adapt to it, e.g. by employing artificial neural networks, pattern recognition and machine learning techniques (Yokoi et al., 2004). Experiments have shown, for instance, that the inclusion of the patient's intention in the control of the electrical stimulation applied to the leg muscles can improve the performance of a paraplegic support system (Riener et al., 2000).

In the context of rehabilitation robotics, this idea was tested by implementing an artificial controller for a functional electrical stimulation support system for paraplegics that considers the whole-body dynamics involved in human walking to predict the next desired 

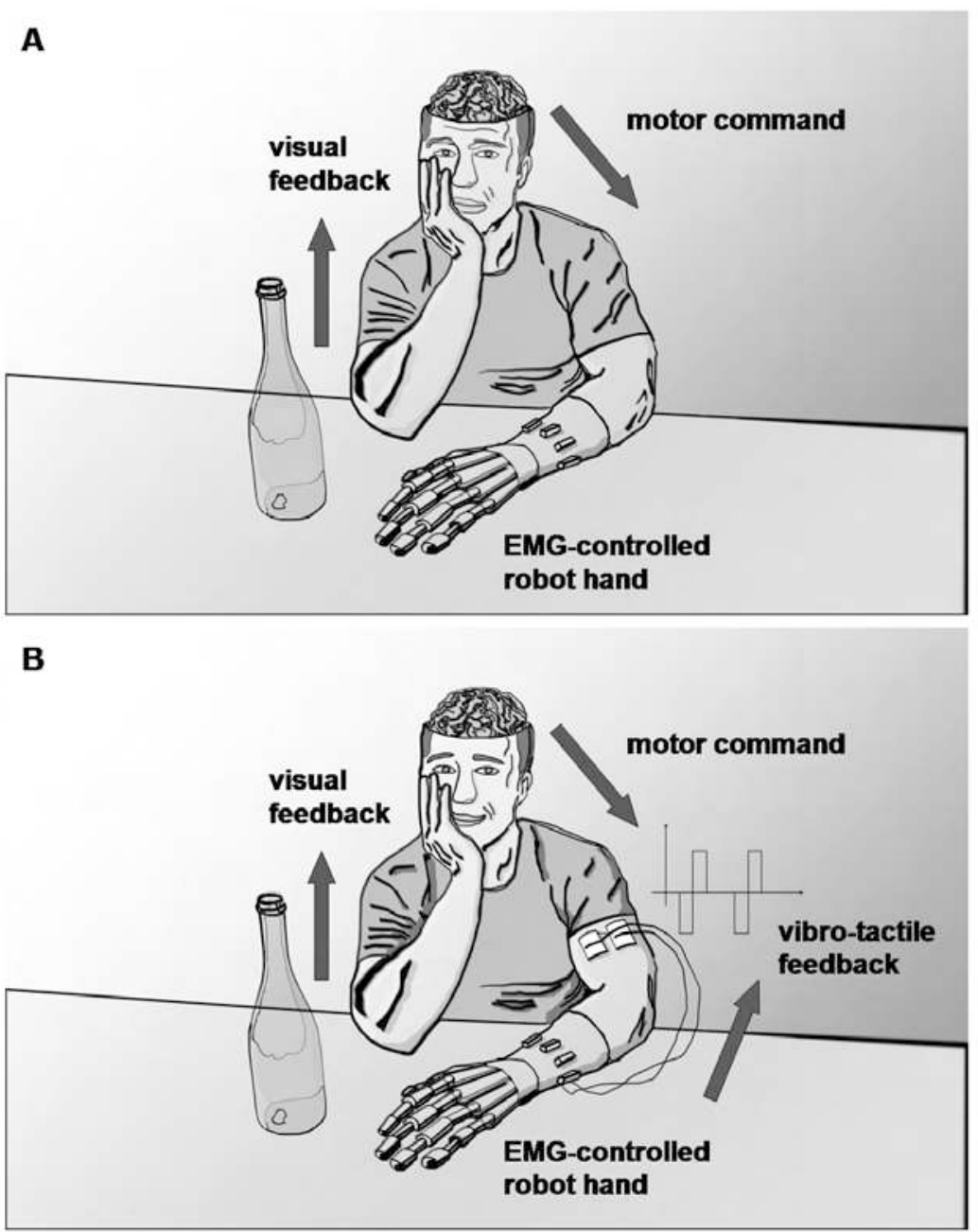

Fig. 1: Disruption of sensory-motor coupling. The disruption of the sensory-motor pathway has a direct effect on the interaction with the environment. (A) A lack of body awareness necessitates an increased cognitive effort to restore control of the body in an adapting environment. (B) The insertion of sensory feedback reduces cognitive effort and improves the interaction with prosthetic devices.

movement (Heliot et al., 2007). The coexistence of artificial and natural controllers allowed the patient some degree of control of otherwise paralyzed limbs. There are two implications from these studies. First, the use of "smart" machines can assist in improving the interaction, but the human brain must be included into the equation - being the most adaptive "machine" at our disposal. Second, in applications in which human and "smart" device interact directly, there is a need for an intuitive communication channel between man and machine. Several studies have addressed the realization of health-related robotic platforms that interact with humans. Ideally, such "human-centered" platforms (Riener et al., 2005; 
Riener, 2007) have to be safe, flexible, mechanically compliant, adaptive towards the user's needs, and easy to use. Moreover, they need to actively involve the patient in the rehabilitation process such that the recovery of lost motor function can be sped up. "Human-centered" strategies have to be contrasted with traditional "controller-centered" approaches in which the patient has to submit to the controller, e.g. a desired reference signal. Our long-term objective is to reduce system complexity by incorporating the plasticity of the human body and brain into the rehabilitation process. Towards achieving this goal, in this chapter, we present a case study based on an EMG-controlled prosthetic hand in which we study the sensory-motor patterns emerging from the interaction between the human, the robot hand, and the surrounding environment. An adaptive learning mechanism attempts to match the movements of the robot hand to those of the user's hand. To address the lack of integration, we apply an electrical stimulation to the user whenever the robot hand touches an object, eliciting a tactile sensation on the user's body. By employing a functional magnetic resonance imaging (fMRI) device, we then evaluate the human-machine interaction looking for the requirements behind the production of the illusion of "body ownership." We test two sensory modalities (visual and tactile) in the manipulation of the robot hand to gain insights into the mechanisms responsible for the extension of the user's body representation. Apart from looking at neural activity in the motor cortex, we also investigate related changes that occur in the sensory cortex.

\section{Prosthetics applications}

In what follows, we analyze the two principal characteristics for the interaction with prosthetic devices: human intention detection and sensory feedback.

\subsection{Human intention detection}

One of the biggest challenges for the field of human-machine interaction is the prediction of the intent of subjects to perform actions such as hand or finger movements. There exist many methods for predicting human movement intention, ranging from the real-time analysis of sensory data (Heliot et al., 2007) to the assessment of biological and physiological signals such as electroencephalograms (EEGs) or electromyograms (EMGs) (Kato et al. 2006, Bitzer \& van der Smagt, 2007). Because of its relevance to the content of this chapter, we only review the use of EMG signals in the context of prosthetics applications.

EMGs are the electrical manifestation of the neuromuscular activity associated with a contracting muscle. Two properties make EMG signals particularly well suited for detecting the intention of movements: (1) EMG signals are directly linked to the desire of movement of a person, whether the movement is executed voluntarily or is initiated through a reflex response; and (2) EMG signals are emitted early, before the muscles contract, and hence can be used for prediction. The problem of intention detection has been tackled by a plethora of scientific work with varying degrees of satisfaction (Wang et al., 2006; Katsis et al. 2005; Nazarpour et al., 2005). Other efforts have focused on the discrimination of a number of hand movements through feature extraction and on improving the effect of a real-time learning for a prosthetic hand (Khezri et al., 2007). Although the accuracy of the recognition is high, these algorithms have not been yet applied to a system that tests their actual efficacy within the demand of a real amputee. In general, feature extraction raises concerns with 
respect to the techniques that select the meaningful data in the context of real world scenarios, where muscles can get tired, and EMG signals are non-stationary. Ways of dealing with the nature of EMG signals in the processing stage rely on the use of different filters and signal processing techniques. One method to transform such signals into an efficient representation is to use families of functions invariant to translation and scaling. By feeding the resulting signals to a neuro-fuzzy classifier, it becomes possible to infer the intention of a person to stand up or sit down (Hussein and Granat, 2002). Support Vector Machines have also been employed for categorizing finger movements. In conjunction with a maximum likeliness measure, the results were sufficiently robust to partition the finger movements in the case of arm pronation (Bitzer et al., 2006).

\subsection{Sensory feedback}

Neurological studies suggest that the self-attribution of body parts is mediated by correlated multisensory feedback (Armel, 2003; Ramachandran et al., 2000). Therefore, when provided with synchronous stimulation, the brain combines the stimuli and associates them to a unique perceptual event. For instance, the sight of brushing of a rubber hand at the same time as brushing of the person's own hand (but hidden from view) is sufficient to produce a feeling of ownership of the fake hand (Ehrson et al., 2005). This illusion of body ownership is called the "rubber-hand illusion" (Constantini \& Haggard, 2007; Ehrson et al., 2005) and seems to originate from the ambiguous but correlated sensory information fed to the brain, which leads to the sensation of having the rubber hand incorporated into the body schema. By using this finding, one could think of fooling the brain into accepting a prosthetic device as an integral part of the body, hence reducing the cognitive effort required for its control. This idea is supported by an experiment conducted on monkeys, which shows that cortical motor neurons that fire when a hand is grasping an object, also fire when the object is grasped with a pair of pliers (Umilta et al., 2008). The outcome of this experiment strongly indicates that the tool is embedded in the monkey's bodily representation as if it would be the monkey's own hand. The aforementioned experiments seem also to suggest that the brain forms internal representations based on the sensory information fed back to it. In this sense, one major drawback of EMG-controlled devices is the minimal or non-existent biofeedback, that is, information on the prosthetic device in relation to the body.

The human body incorporates a robust and redundant sensory system, by which if a part fails, nearby ones are used in order to restore the lost function. The user of a prosthetic device usually needs to overcome the lack of tactile and proprioceptive data with visual feedback, which increases the cognitive effort required to control the device (Weir, 1998). This conscious effort is one of the main reasons that amputees abandon the use of current myoelectric devices (Biddiss, 2007). We conclude that prosthetic devices need to include a feedback source that enables the user to extend his physiological proprioception (Simpson, 1974). Such sensory feedback is of course a prerequisite not limited only to prosthetic applications; spinal-cord injury patients also share the same requirement.

Methods for providing feedback to the human body can be classified into two categories: invasive and non-invasive. Invasive methods directly stimulate nerve fibers to transmit sensations to the brain. For example, Shimojo et al. (2003) inserted electrodes in the nerve axons of a person that then were used to transmit tactile information sampled from a 
robot. More recently, Dillon et al. (2005) implemented a controller for a robot hand that used both afferent and efferent neural paths for communication from and to the human body, i.e. for controlling the robot hand and for receiving tactile feedback from the robot platform. Non-invasive methods are more widespread in their implementations. The most common method to transmit information to the body is transcutaneous (surface-mounted) electrical stimulation (Back-y-Rita et al., 2003; Kaczmarek et al., 2006). There is also relevant research looking for appropriate stimulation areas in the human body for data transmission (Riso, 1999); as well as the modulation used to increase the amount of data transmitted into the body (Kaczmarek, 2000; Kim, 2005). Besides electrical stimulation, mechanic vibrators are an additional option for data transmission. Although they are typically used in haptic interfaces (Honma et al., 2004), they find also application in the context of prosthetics (RiosPoveda, 2002).

\section{Experimental setup}

For our experiments, we used a prosthetic hand controlled through EMG signals (Yokoi et al., 2004). The raw EMG signals were processed producing a set of feature vectors that in turn were used to generate a database of intended motions. Feedback to the user of the hand was provided through transcutaneous functional electrical stimulation (Szeto, 1992; Kackzmareck, 2006). In what follows, we give an overview of the parts composing the experimental setup. First, we describe the prosthetic hand and the EMG-based intention detection system. Then, we present the visuo-tactile feedback system. Finally, we expose the fMRI scan setup and the related data analysis.

\subsection{Prosthetic hand}

The EMG-controlled prosthetic "humanoid" hand employed in this study was composed of five fingers and had 13 degrees-of-freedom (DOF) (Hernandez-Arieta et al., 2006a). Each finger had three joints and two DOFs, the distal-inter-phalangial joint (DIP) and the proximal-interphalangial joint (PIP) were actuated by the same tendon, and the metacarpal (MP) joint was actuated by a single tendon. The wrist and the MP joint of the thumb control used two motors for the actuation of pronation/supination and extention/flection movements. The robot hand had pressure sensors placed over the PIP joint of fingers, on the fingertips, and in the palm. Force sensing resistor (FSR) based pressure sensors - due to their flexibility and ease of installation - were used to detect the interaction with the environment. EMG signals were detected by surface electrodes placed at muscle sites on the residual limb. The raw signals were processed and eventually used to control the robot hand.

\subsection{EMG signals and classification}

For the EMG patterns classification, we used a feed-forward neural network with an automatic learning mechanism (Kato et al., 2006). The EMG pattern classification system is composed of three units (Fig. 3): (a) an analysis unit, (b) a classification unit, and (c) a supervision unit. The analysis unit is in charge of extracting feature vectors from the raw EMG signals. 


\section{Classification Process}

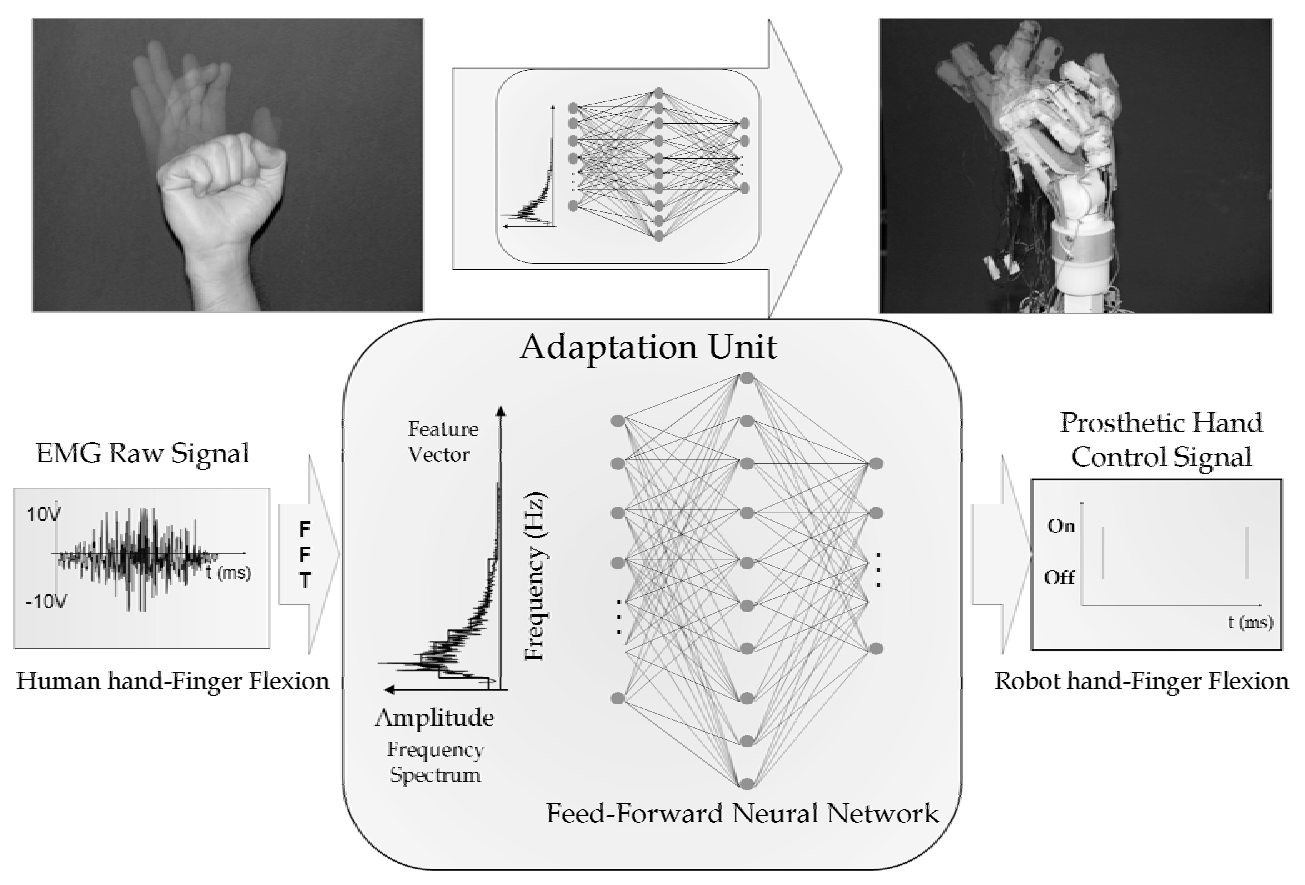

Fig. 2 EMG classification process. Raw EMG data is converted into a set of feature vectors that are fed into the ANN for classification. The ANN is trained to map each feature vector into a hand configuration.

The unit performs a Fast Fourier Transform of the acquired EMG signals producing a power density spectrum. We extract the feature vector from the power spectrum of all the EMG sensors. For this study, the feature vector extracts 8 samples from 3 EMG channels. The classification unit is in charge of generating clusters for the recognition of several hand movements. It consists of a supervised feed-forward artificial neural network with back propagation for the calculation of the weights. The supervision unit provides the system parameters for the evaluation of the feature vectors in the classification unit. Once a feature vector has been identified, the unit generates a control command to produce the desired robot hand movement. The supervision unit evaluates and updates the classification unit until the system achieved the expected motion, looking for the mapping function that denotes the relationship between the feature vectors and the expected robot hand motion. It receives 16 feature vectors for each motion that was included into the feature vectors' database.

\subsection{Visuo-tactile feedback}

We used a functional electrical stimulation device (Hernandez-Arieta, 2006b) to provide feedback by directly stimulating the skin mechanoreceptors. The produced 


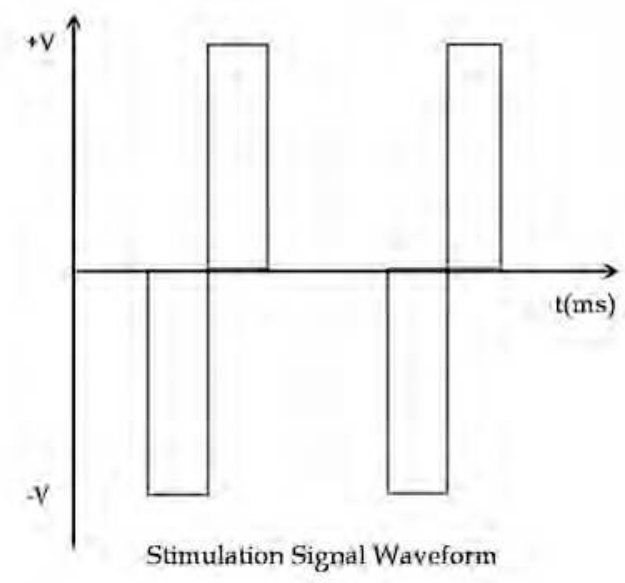

Fig. 3 Electrical stimulation waveform. The negative part of the signal depolarizes the nerves' axons, promoting vibro-tactile sensation. The positive charges avoid tissue damage by eliminating charge accumulation.

electrical signal follows the guidelines given from previous applications of electrical stimulation in prosthetic applications that define the required frequency, voltage and waveform shape (Pfeiffer, 1968; Melen \& Meindl, 1971; Szeto, 1992). A high frequency, biphasic signal is efficient in interacting with the sensory system (Fig. 3). To regulate the intensity of the stimulation and to avoid damaging the skin (due to excessive charge accumulation), the duty rate of the positive and negative phases of the signal changed simultaneously, while the frequency was kept constant (Grill \& Mortimer, 1995).

Like other sensory systems, mechanoreceptors habituate to constant stimuli. Hence, we set the stimulation intensity to be strong enough to be detected by the skin mechanoreceptors, while being weak enough to not stimulate noxious (pain) receptors and muscle fibers. Figure 4 presents the experimental concept. Whenever the robot hand touches an object, the pressure sensors located in the hand are used to electrically stimulate the user, providing tactile feedback. Because the person can in addition see the robot hand's movements, there is also visual feedback.

\section{4. $\quad$ fMRI scan setup}

To accommodate visual feedback, the robotic hand has to be within the line of sight of the user. However, the fMRI apparatus involves strong magnetic forces, prohibiting the placement of the robot hand within the user's visible area. To overcome this difficulty, a video camera, in conjunction with a set of mirrors, was used to project the prosthetic hand and its surrounding environment within the fMRI room (Figure 4). The EMG sensors and the stimulation electrodes were coated with aluminum foil to shield them from magnetic fields. The EMG sensors were then placed on the right forearm, while the electrical stimulation electrodes were placed on the upper left arm of the subjects. This was done to reduce the effects of the electric stimulation over the EMG acquisition process. The volume acquisition was done with a 1.5T MAGNETOM Vision plus MR scanner (Siemens, Erlangen, 


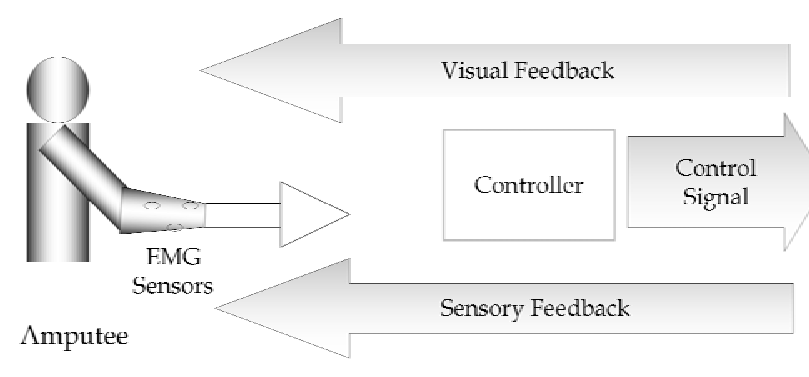

Neurostimulation Electrodes

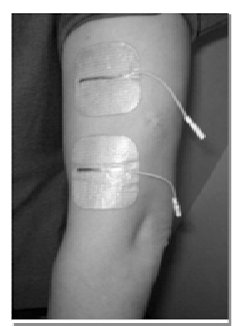

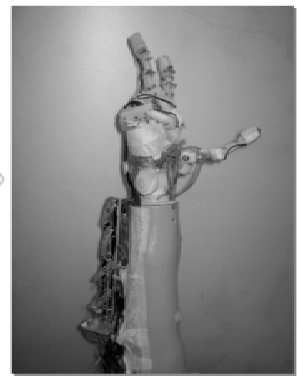

Robot Hand

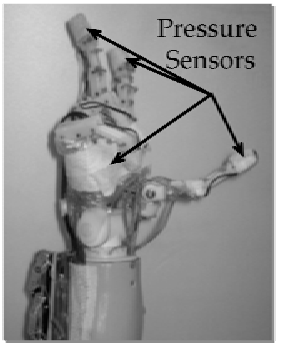

Fig. 4 Experimental setup. Top: the participant receives both visual and tactile feedback from the robotic platform. Bottom: tactile information derived from the pressure sensors is used to provide electrical stimulation to the left upper arm of the user of the device.

Germany) using the standard head coil. We used foam padding around the subjects' head to minimize head motion and discarded the first five volumes of each fMRI scan because of non-steady magnetization; the analysis was performed using the remaining 54 scans. The fMRI protocol was a block design with one epoch for the task and rest conditions. Each epoch lasted 24 [s] which is equivalent to three whole-brain fMRI volume acquisitions. We used the Statistical Parametric Mapping software 2 (Holmes, 1994) for the analysis of the data. The duration of the first type of experiment (see Section 4) was 5 [s], with a scan time of 3 [s] and a rest time of 2 [s] between scans and we acquired 4 sessions of 35 scans. In the second type of experiment (Section 4) the duration for one scan was 7 [s], with a scan time of 3 [s] and a rest time of 4 [s] between scans; we performed two sessions of 35 scans each. We used an echo-planar imaging (EPI) template to realign the scans and transform them into the standard stereotactic space of Talairach (Talairach, 1988). Data was then smoothed in a spatial domain (full width at half-maximum $=8 \times 8 \times 8[\mathrm{~mm}]$ ) to improve the signal-to-noise ratio. After specifying the appropriate design matrix, the delayed box-car function as a reference waveform, the condition, and the slow hemodynamic fluctuation unrelated to the task, the subject effects were estimated according to a general linear model taking temporal smoothness into account. We then applied a proportional scaling for the global normalization. Once this process was completed, we compared the estimates using the linear contrasts of rest and task period to test the hypotheses about regionally specific condition effects. The resulting set of voxel values for each contrast constituted a statistical parametric map of the $t$ statistic, $\operatorname{SPM}(t)$. For the analysis of each session, we assigned a 


\section{fMRI Room Setup}

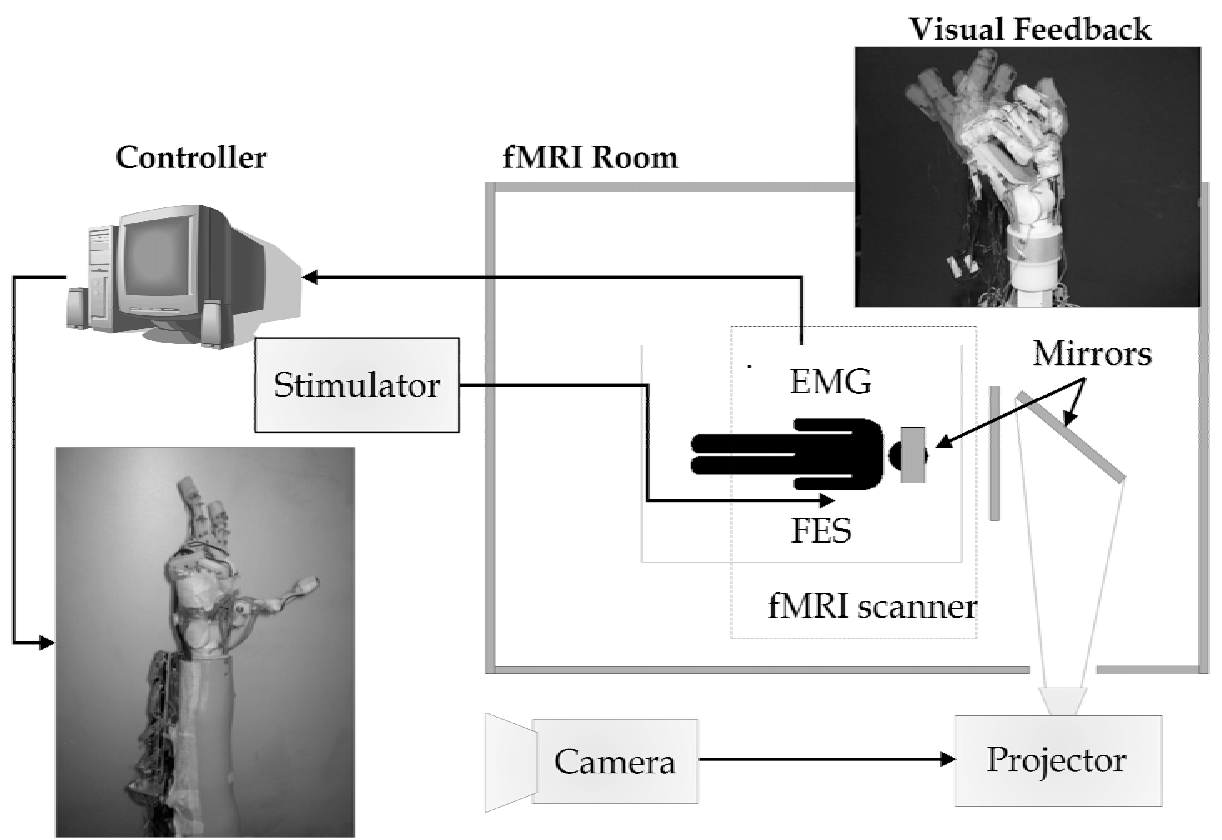

Fig. 5 fMRI room setup. The robot hand is placed outside the fMRI scanner room. A video camera records the robot hand whose movement is then projected through a set of mirrors inside the fMRI scanner.

threshold of $\mathrm{P}<0.001$ to the voxels and significant clusters, which were not corrected for multiple comparisons. The minimum threshold for comparison was set to $\mathrm{T}=2.9$ for passive application of electrical stimulation, and $\mathrm{T}=3.26$ for the active manipulation of the robot hand.

\section{Experiments}

In order to evaluate the effects of the interaction between the sensory and motor modalities during the function recovery, we conducted "passive" and "active" experiments. In the passive experiments, we applied an electrical stimulation to the subjects irrespectively of the configuration of the robot hand. Concurrently, we measured the subjects' cortical activation response.

We conducted two types of active experiments, one in which there was only tactile feedback and one in which there was both visual and tactile feedback. The first type aimed at providing insight into the relationship between the subjects' sensory and motor areas without the use of visual feedback. We asked the subjects to open and close the fingers of 
the robot hand. The neural network processing the EMG signals was trained individually for each participant using a finger flexion/extension motion. A ball was periodically placed inside the robot hand. Every time the subjects grabbed the ball with the hand, the pressure sensors located at the fingertip triggered the electrical feedback process, stimulating the subject's upper left arm. To evaluate the multimodal sensory-motor relationship, visual feedback was added. The subjects were shown an object and were asked to grasp it using the robot hand. The movements of the hand as well as its surrounding environment were projected to the visual field of the user using the method described in Section 3.4.

Three participants took part in our experiments: one test subject and a control group composed of two healthy participants. The test subject was a woman in her 50's with a right arm amputation performed 5 years before this study. As her amputation was above the wrist level, the majority of the forearm was intact. The control group consisted of two healthy men in their 20's, with no visible physical abnormalities. To avoid biased results, for all participants three EMG sensors were placed in locations focusing on the major muscle groups on the forearm: extensor digitorums, flexor digitorum superficialis, and flexor digitorum profundus. To measure the effect of the continuous use of the robot hand, the amputee patient was asked to use the robot hand on a daily basis over a period of three months. fMRI scans were taken at the beginning of the study, one month after, and at the end of the study. After each session, the subjects had to fill in a questionnaire regarding the sensations perceived during the tasks.

\section{Results}

At the beginning of each experimental session, in order to establish a common ground for the active experimentation that followed, all subjects were subjected to passive electrical stimulation. The results are presented in Figure 6, using a base T value of 2.9 for comparison. Passive electrical stimulation without manipulating the control hand showed no activation of the sensory and motor cortices (Brodmann areas $3 \& 4$ respectively) in either hemisphere. However, the parietal area of both hemispheres, responsible for processing sensory data, did present mild activation. At the end of the three-month experimentation period and using the robot hand on a daily basis, an apparent reversion of the cortical reorganization process in the amputee's brain can be observed (Figure 5). The results obtained from the fMRI portray a major reduction of cortical activity in the general area of the brain. The parietal area and motor cortex present a significant activation reduction. The answers both the amputee and the control subjects provided in the questionnaire clearly indicate the "rubber-hand illusion"; they were feeling as if their actual right hand was touching the presented object. The results from the control subjects however show a more specific activation of the motor cortex (Brodmann Area 4) than that in the amputee's case. This is speculated to relate to the amputees' unused lost limbs, not utilized in daily life activities. In addition, the lack of sensory input from a lost limb results in a cortical reorganization process occurring after an amputation, essentially recycling the no longer active cortical regions for using on other tasks. Using a base $\mathrm{T}$ value of 3.6, the control subjects presented activation of Brodmann area 4 and Brodmann area 3 in the left hemisphere of the brain for the manipulation of the robot hand with tactile and visual feedback. There was no visible activation of Brodmann area 3 in the right hemisphere. To identify the influence of the visual sensory modality on the generation of tactile illusions, visual feedback was 


\section{Passive Electrical Stimulation}

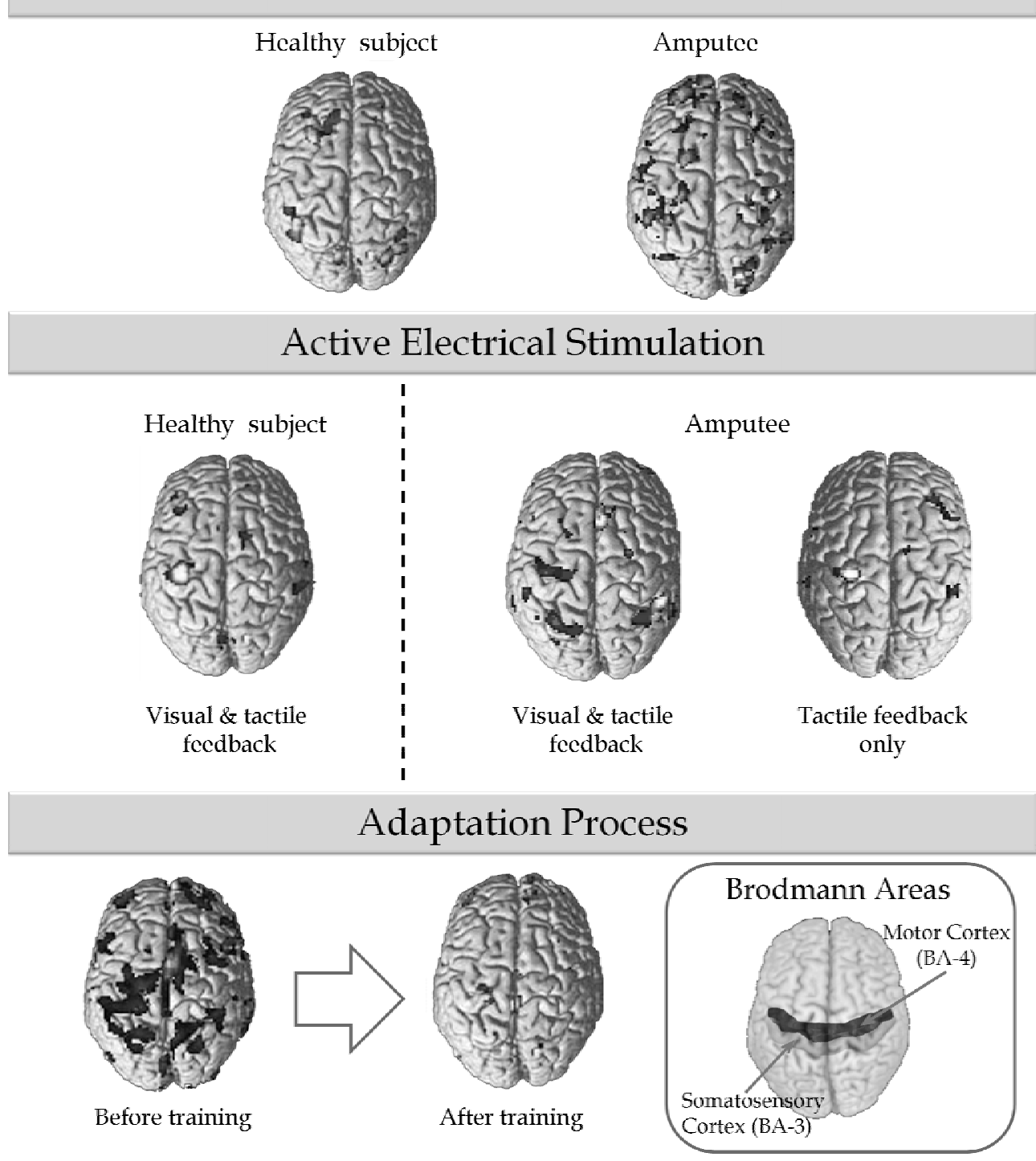

Fig. 6: Experimental results. Top: Passive electrical stimulation shows no activation of Brodmann area 3 for the healthy subject. The amputee presents cortical reorganization with light broad activation. Center: The healthy subject presents no activation of the right hemisphere for active manipulation of the robot hand with multisensory feedback. The amputee presents no activation of the right hemisphere for both multisensory feedback and tactile feedback. Bottom: After three months of use, the amputee's neural activity displays reversion of cortical reorganization. The amputee's brain presents less activation in Brodmann areas $3 \& 4$. 
suppressed. Figure 6 shows the results from applying electrical stimulation in the left upper arm of the patient without visual feedback. The comparison between the usual stimulation with visual feedback shows an increase in the somatosensory area (Brodmann Area 3). The motor cortex exhibits the same level of activation as with the included visual feedback experiments. The activation for the case with visual feedback had a $T$ value of 4.49 for MNI coordinates $x=-30, y=-26, y=52$. In absence of visual feedback, the $T$ value was 6.48 for MNI coordinates $x=-24, y=-26, z=52$. In the blind case, the right hemisphere presented activation of Brodmann area 3 at MNI coordinates $x=24, y=-30, z=51$. The continuous use of the robot hand with tactile feedback during a period of three months led to a reduction of the cortical reorganization that the patient suffered after amputation.

\section{Discussion}

It is interesting to observe how the brain reacts when stimulation occurs as an isolated event during the passive experiments. In such a case, the parietal lobe "perceives" the stimulation as a source of pain or as an uncorrelated stimulus presented to the body. However, when electrical stimulation is used for grasping an object, there are three channels that work in synergy; the intention of the subject, the visual feedback, and the stimulus provided by the electrical stimulation. This sequence of events takes place several times during the scanning process, allowing the brain to combine the various pieces of information as a single, simultaneous and repetitive event. The fMRI results provide an indication of how the brain adapts to accommodate the perception induced from electrical stimulation applied on the left arm. During active experimentation, the primary area in the somatosensory cortex (Brodmann Area 3) which is related with the left arm points to an increased activation, sufficient to register changes in the fMRI data. The brain clearly identifies the stimulation as taking part on the left arm. However, comparing these results to the ones obtained during the passive experimentation, we find additional areas of increased activation, not present during the passive tests. The sensory area associated with the right hand is activated, suggesting that the brain is correlating the multi-sensory input as a single event, localizing it in the right hand (in this case, the prosthetic hand). The subjects do not interact directly with the object in question; rather, they do so through the robot hand. Such results clearly indicate the role of brain plasticity in the creation of new communication channels between the user of a robotic device, and the device itself. fMRI measurements proved to be useful and reliable for objectively measuring changes in cortical activation while using the robotic platform, allowing for detailed feedback on the workings of the subjects' brain. The removal of visual feedback as a sensory channel during experimentation leads to two interesting observations: (1) the influence of visual feedback on the illusion of ownership decreased over extended periods of use; and (2) tactile habituation alone is enough for generating the illusion of ownership of the robot hand. The use of myoelectric prostheses acts against the cortical reorganization process that takes place after an amputation. The simultaneous application of electrical stimulation along with an adaptive prosthetic system that adheres to the user's intention promotes the generation of the illusion of ownership; the amputee is able to reconstruct their impaired body image by incorporating the prosthesis. In this respect, a particular emphasis should be given to the nature of the artificial signals provided to the human body. They should be shaped in such fashion that they promote the inclusion of the prosthetic system in the user's body schema. 
Electrical stimulation has proven to be a feasible way to accomplish this goal, reinforcing the vigor of the involved muscles and, in consequence, shaping the body schema. All these results open the possibility to develop novel man-machine interfaces that allow for the subconscious control of an external (e.g. prosthetic) device.

\section{Conclusions}

To answer the question as to what extent the sensory and motor function are interoperating during rehabilitation, we presented fMRI data on apparent brain activation during the use of both motor and sensory modalities. An EMG-controlled prosthetic hand provided the necessary motor function recovery, and an electrical signal was utilized for tactile feedback.

The inclusion of tactile feedback while actively controlling the EMG prosthetic device produced a tactile illusion in the left hemisphere of the patient's brain. This result is surprising given that we applied the stimulation to the opposite arm. The production of the tactile illusion points to a correlative behavior of the brain in processing active motor commands and their corresponding feedback. To test the conditions for the generation of the tactile illusion, we performed both passive and active administrations of electrical stimulation, where the interaction of the robot hand with an object triggered the active administration of electrical stimulation. We tested the influence of the different sensory modalities by removing visual feedback from the setup.

We confirmed the presence of the tactile illusion in all tests except for the passive administration of electrical stimulation. These experiments serve to demonstrate how the active involvement of disabled individuals improves the sensory-motor function recovery by effectively promoting the correlation process in the brain. Of equal importance, the illusion of ownership is only dependant on tactile feedback with or without the inclusion of visual feedback. Our findings point to a flexible structure that can be modified if we provide adequate feedback within an adequate period.

Future work will aim at contributing more to the human-centered approach to rehabilitation. The results concerning the interaction of motor and sensory modalities for function recovery are already promising; nevertheless, it is imperative to test a larger number of patients. Finally, intercepting the sensory-motor interaction between human and prosthetic devices allows an investigation into ways of improving and shaping it. Linked with the need of an amputee, such an approach bears the importance of relieving the user from a steady conscious mental activity in order to manipulate the prosthetic device.

\section{References}

Adee, S. (2008). Dean Kamen's "Luke Arm" Prosthesis Readies for Clinical Trials, IEEE Spectrum, February.

Atkins, D.J., Heard. D.C.Y., \& Donovan, W.H. (2001). Epidemiologic overview: Individuals with upper limb loss, and their reported research priorities. Int. J. of Prosthetics and Orthotics, 25: 228-227.

Au, S.K., Bonato, P., \& Herr, H. (2005). An EMG-position controlled system for an active ankle-foot prosthesis: an initial experimental study. Proc. of 9th Int. Conf. on Rehabilitation Robotics, pp. 375-379. 
Bach-y-Rita, P., Tyler, M.E., \& Kaczmarek, K.A. (2003). Seeing with the brain. Int. J. of Human-Computer Interaction, 15(2): 285-295.

Biddiss, E., Beaton, D., \& Chau, T. (2007). Consumer design priorities for upper limb prosthetics. Disability and Rehabilitation: Assistive Technology, 2(6): 346-357.

Bitzer, S. \& van der Smagt, P. (2006). Learning EMG Control of a robotic hand: Towards Active Prostheses. Proc. of 2006 IEEE Int. Conf. on Robotics and Automation, pp. 28192823.

de Castro, M. C. \& Cliquet, A. (2000). Artificial sensorimotor integration in spinal cord injured subjects through neuromuscular and electrotactile stimulation. Artificial Organs, 24 (9): 710-717.

Dhillon, G.S., Horch, K.W., \& DeBakey, M. E. (2005). Direct neural sensory feedback and control of a prosthetic arm. Neural Systems and Rehabilitation Engineering, ISSN1534-4320, 13 (14): 468-472.

Dollar, A.M. \& Howe, R.D. (2007). The SDM hand as a prosthetic terminal device: a feasibility study. Proc. of the 2007 IEEE Int. Conf. on Rehabilitation Robotics..

Grill, W. M. \& Mortimer, T. (1995). Stimulus waveforms for selective neural stimulation. IEEE Engineering in Medicine and Biology, 14(4): 375-385.

Hacking, H.G.A, Berg, J.P., Van Der Dahmen, K.T., \& Post, M.W.M. (1997). Long term outcome upper limb prosthetic use in the Netherlands. Eur. J. of Physical Medicine and Rehabilitation, 7: 179-181.

Heliot, R., Azebedo, C., \& Espiau, B. (2007). Rehabilitation Robotics, Sashi S. Kommu (ed.), Itech Education and Publishing, Vienna, Austria, ISBN 978-3-902613-04-2, pp: 163186.

Henrik-Ehrsson, H., Holmes, N., \& Passigham, R. (2005). Touching a rubber hand: feeling of body ownership is associated with activity in multisensory brain areas. J. of Neuroscience, 25(45): 10564-10573.

Henrik-Ehrsson, H., Wiech, K., Weiskopf, N., Raymond, J. D., \& Passingham, R. E. (2007). Threatening a rubber hand that you feel is yours elicits a cortical anxiety response. Proc. Nat. Acad. of Sciences USA, 104(23): 9828-9833.

Hernandez-Arieta, A., Kato, R., Yokoi, H., Yu, W., (2006a). Development of a multi-DOF electromyography prosthetic system using the adaptive joint mechanism. Applied Bionics and Biomechanics, 3(2): 101-111.

Hernandez Arieta, A., Yu, W., Yokoi, H., Arai, A. (2006b). fMRI study of the cross-modal interaction in the brain with an adaptable EMG prosthetic hand with biofeedback, Proc. of the 28th Ann. Int. Conf. of IEEE Eng. In Med. and Biol. Soc., pp: 1280-1284.

Holmes, A.P. (1994). Statistical Issues in Functional Brain Mapping. PhD Thesis, University of Glasgow.

Hunter, J.P., Katz, J., \& Davis, K.D. (2003). The effect of tactile and visual sensory inputs on phantom limb awareness. Brain, 126(3): 579-589.

Kaczmarek, K.A., Nammi, K., Agarwal, A.K., Tyler, M.E., Haase, S.J., \& Beebe, D.J. (2006). Polarity effect in electrovibration for tactile display. IEEE Trans. on Biomed. Eng., 53(10): 2047-2054.

Kaczmarek, K. A.. (2000). Electrotactile adaptation on the abdomen: preliminary results. IEEE Trans. Rehabil. Eng., 8(4): 499-505. 
Kato, R., Yokoi, H., \& Arai, T. (2006). Competitive learning method for robust EMG-toMotion Classifier. Proc. of Conf. on Intelligent Autonomous Systems 9, IOS Press, ISBN 1-58603-595-9

Kim, G., Asakura, Y., Okuno, R., \& Akazawa, K. (2005). Tactile substitution system for transmitting a few words to a prosthetic hand user, Conf. Proc. IEEE Eng. Med. Biol. Soc., 7,pp: 6908-6911.

Lancaster, J. L., Woldor, M. G., Parsons, L. M., Liotti, M., Freitas, C. S., Rainey, L., Kochunov, P. V., Nickerson, D., Mikiten, S. A., \& Fox, P. T. (2000). Automated talairach atlas labels for functional brain mapping. Human Brain Map, 10( 3):120-131

Kuyper, M.A., Breedijk, M., Muldres, A.H.M, Post, M.W.M, \& Prevo, A.J.H. (2001). Prosthetic management of children in the Netherlands with upper limb deficiencies. Prosthetics and Orthotics International, 25: 228-237

Lebedev, M. A., \& Nicolelis, M.A.L. (2006). Brain-machine interfaces: past, present and future, Trends Neuroscience, 29( 9): 536-546

Lotze, M., Grodd, W., Birbaumer, N., Erb, M., Huse, E., \& Flor. H. (1999). Does use of a myoelectric prosthesis prevent cortical reorganization and phantom limb pain? Nature Neuroscience, 2(6): 501-502

Lotze, M., Montoya, P., Erb, M., Hulsmann, E., Flor, H., Klose, U., Birbaumer, N., \& Grodd W. (1999). Activation of cortical and cerebellar motor areas during executed and imagined hand movements: an fmri study. J Cognitive Neuroscience, 11(5)pp: 491-501

Lotze, M., Flor, H., Grodd, W., Larbig, W., \& Birbaumer, N. (2001). Phantom movements and pain. An fmri study in upper limb amputees. Brain, 124(11): 2268-2277

Logothetis, N. K. (2002). The neural basis of the blood-oxygen-level-dependent functional magnetic resonance imaging signal. Phil. Trans. Real Soc. London B| Biological Sciences, 357(1424): 1003-1037

Melen, R. D. \& Meindl, J. D. (1971). Electrocutaneous stimulation in a reading aid for the blind. IEEE Trans Biomed Eng, 18( 1) :1-3

Pappas, I. P., Popovic, M. R., Keller, T., Dietz, V., \& Morari, M. (2001). A reliable gait phase detection system. IEEE Trans. Neural Sys. Rehabil. Eng., 9 ( 2): 113-125

Pfeifer, R., Lungarella, M. and Iida, F. (2007). Self-organization, embodiment, and biologically inspired robotics. Science, 318: 1088-1093.

Pfeifer, R., \& Bongard, J. C. (2006). How the Body Shapes the Way We Think: a New View of Intelligence (Bradford Books). The MIT Press, ISBN:0262162393

Pfeiffer, E. A. (1968). Electrical stimulation of sensory nerves with skin electrodes for research, diagnosis, communication and behavioral conditioning: a survey. Med. Biol. Eng., 6( 6):637-651, November

Plettenburg, D.H. (1998). Basic requirements for upper extremity prostheses: the wilmer approach. In H.K. Chang and Y.T. Zhang, editors, Proceedings of the 20th Annual International Conference of the IEEE Engineering in Medicine and Biology Society, ISBN 0-7803-5164-9, volume Vol. 5/6, pp: 2276-2281

Pylatiuk, C., \& Doderlein, L. (2006). "Bionic" arm prostheses. State of the art in research and development, Orthopade, 35: 1169-70, 1172-5

Ramachandran, V.S., \& Rogers-Ramachandran, D. (2000). Phantom limbs and neural plasticity. In Archives Neurology, 57: 317-320

Rios-Poveda, A. (2002). Myoelectric prosthesis with sensorial feedback. In University of New Brunswick MyoElectric Controls/Powered Prosthetics Symposium 
Riso, R. R., Ignagni, A. R., \& Keith, M. W. (1991). Cognitive feedback for use with fes upper extremity neuroprostheses. IEEE Transactions Biomedical Engineering, 38(1):29

Riso, R. R.(1999). Strategies for providing upper extremity amputees with tactile and hand position feedback--moving closer to the bionic arm. Technol Health Care, 7:401-409

Riener, R., Ferrarin, M., Pavan, E. E., \& Frigo, C. A. (2000). Patient-driven control of fessupported standing up and sitting down: experimental results. IEEE Transactions in Rehabilitation Engineering, 8(4):523-529

Riener, R., Frey, M., Bernhardt, M., Nef, T., \& Colombo, G. (2005). Human-centered rehabilitation robotics. Proc. of the 2005 IEEE 9th Int. Conf. on Rehabilitation Robotics

Riener, R. (2007). Robot-aided rehabilitation of neural function in the upper extremities, Acta Neurochir Suppl, 97 (1): 465-471

Rorden, C., \& Brett, M. (2000). Stereotaxic display of brain lesions. Behav Neurol, 12(4):191200

Sabolich, J.A., \& Ortega, G.M. (1994). Sense of feel for lower-limb amputees: A phase one study. Journal of Prosthetics and Orthotics, 6: 36-41,

Szeto, A. Y. \& Farrenkopf, G. R. (1992). Optimization of single electrode tactile codes. Annals of Biomedical Engineering, 20 (6): 647-665

Simpson, D.C. (1974). The choice of control system for the multimovement prosthesis: Extended physiological proprioception (e.p.p.). In P. Herberts, R. Kadefors, and R. Magnussonand I. Petersen, editors, The Control of Upper-Extremity Prostheses and Orthoses. Springfield, Charles Thomas

Scott, R. N. (1990). Feedback in myoelectric prostheses. Clin Orthop Relat Res, (256):58-63

Shimojo, M., Suzuki, T., Namiki, A., Saito, T., Kunimoto, M., Makino, R., Ogawa, Hi., Ishikawa, M., \& Mabuchi, K. (2003). Development of a system for experiencing tactile sensation from a robot hand by electrically stimulating sensory nerve fiber. In 2003 IEEE Int. Con. on Robotics and Automation, pp: 1264-1270

Strother, S. C. (2006). Evaluating fmri preprocessing pipelines. IEEE Eng. in Med. and Bio. Magazine, 25( 2): 27-41

Talairach, J. \& Tournoux, P. (1988). Co-Planar Stereotaxic Atlas of the Human Brain: 3dimensional Proportional System: an Approach to Cerebral Imaging. Thieme Medical Publishers, Inc., ISBN 0865772932

Tsakiris, M., Bosbach S. \& Gallagher, S. (2007). On agency and body-ownership: Phenomenological and neuroscientific reflections. Consciousness and Cognition. 16 (3): 645-60.

Veltman, D., \& Hutton, C. (2000). Spm99 manual. Technical report, Wellcome Department of Imaging Neuroscience

Weir, R.F., Childress, D.S., \& Licameli, J.N. (1998). Motor Prostheses and the Lack of Physiologically Appropriate Feedback: Their function and connections with Manipulators, Robots and other Human-Machine Systems, chapter Section 3.21. MIT Press

Yokoi, H., Hernandez Arieta, A., Katoh, R., Yu, W., Watanabe, I., \& Maruishi, M. (2004). Mutual Adaptation in a Prosthetics Application, Embodied Artificial Intelligence edited by Fumiya Iida, Rolf Pfeifer, Luc Steels, and Yasuo Kuniyoshi, LNCS/LNAI series of Springer 


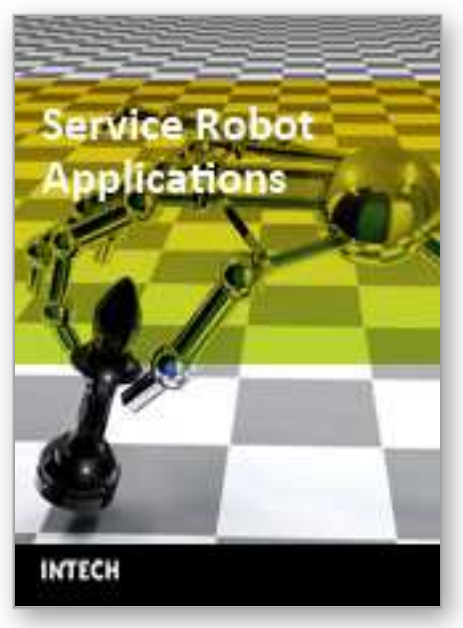

\author{
Service Robot Applications \\ Edited by Yoshihiko Takahashi
}

ISBN 978-953-7619-00-8

Hard cover, 400 pages

Publisher InTech

Published online 01, August, 2008

Published in print edition August, 2008

The aim of this book is to provide new ideas, original results and practical experiences regarding service robotics. This book provides only a small example of this research activity, but it covers a great deal of what has been done in the field recently. Furthermore, it works as a valuable resource for researchers interested in this field.

\title{
How to reference
}

In order to correctly reference this scholarly work, feel free to copy and paste the following:

Alejandro Hernandez-Arieta, Konstantinos Dermitzakis, Dana Damian, Max Lungarella and Rolf Pfeifer (2008). Sensory-Motor Coupling in Rehabilitation Robotics, Service Robot Applications, Yoshihiko Takahashi (Ed.), ISBN: 978-953-7619-00-8, InTech, Available from:

http://www.intechopen.com/books/service_robot_applications/sensorymotor_coupling_in_rehabilitation_robotics

\section{INTECH}

open science | open minds

\section{InTech Europe}

University Campus STeP Ri Slavka Krautzeka 83/A 51000 Rijeka, Croatia Phone: +385 (51) 770447

Fax: +385 (51) 686166 www.intechopen.com

\section{InTech China}

Unit 405, Office Block, Hotel Equatorial Shanghai No.65, Yan An Road (West), Shanghai, 200040, China 中国上海市延安西路 65 号上海国际贵都大饭店办公楼 405 单元 Phone: +86-21-62489820

Fax: $+86-21-62489821$ 
(c) 2008 The Author(s). Licensee IntechOpen. This chapter is distributed under the terms of the Creative Commons Attribution-NonCommercial-ShareAlike-3.0 License, which permits use, distribution and reproduction for non-commercial purposes, provided the original is properly cited and derivative works building on this content are distributed under the same license. 